\title{
CO Vibration as a Probe of Ligand Dissociation and Transfer in Myoglobin
}

\author{
T. Polack, ${ }^{1}$ J. P. Ogilvie, ${ }^{1}$ S. Franzen, ${ }^{2}$ M. H. Vos, ${ }^{1}$ M. Joffre, ${ }^{1}$ J.-L. Martin, ${ }^{1}$ and A. Alexandrou ${ }^{1}$ \\ ${ }^{1}$ Laboratoire d'Optique et Biosciences, UMR CNRS 7645, INSERM U451, Ecole Polytechnique, ENSTA, F-91128 Palaiseau, France \\ ${ }^{2}$ Department of Chemistry, North Carolina State University, Raleigh, North Carolina 27695, USA
}

(Received 17 April 2003; published 1 July 2004)

\begin{abstract}
We report femtosecond visible pump, midinfrared probe, spectrally integrated experiments resolving the dynamics of $\mathrm{CO}$ in myoglobin upon photodissociation. Our results show a progressive change in absorption strength of the $\mathrm{CO}$ vibrational transition during its transfer from the heme to the docking site, whereas the vibrational frequency change is faster than our time resolution. A phenomenological model gives good qualitative agreement with our data for a time constant of $400 \mathrm{fs}$ for the change in oscillator strength. Density-functional calculations demonstrate that indeed vibrational frequency and absorption strength are not linearly coupled and that the absorption strength varies in a slower manner due to charge transfer from the heme iron to $\mathrm{CO}$.
\end{abstract}

DOI: 10.1103/PhysRevLett.93.018102

PACS numbers: 87.64.Je, 33.70.-w, 42.65.Re

The binding of diatomic ligands $\left(\mathrm{O}_{2}, \mathrm{NO}\right.$, or $\left.\mathrm{CO}\right)$ to heme proteins is an integral part of many biological functions such as storage and ligand transport, enzymatic catalysis, and ligand detection. A thorough knowledge of the mechanisms underlying ligand transfer from the heme binding site to the protein exterior is essential toward understanding how these proteins accomplish their function: discriminate between ligands, shuttle them from the solvent to the heme pocket or vice versa, and react to external signals.

Myoglobin ( $\mathrm{Mb}$ ) is an extensively studied heme protein, and the CO ligand embedded in myoglobin is well characterized in both its state bound to the central $\mathrm{Fe}$ atom of the heme and in the "docking site," the first site the ligand reaches on its way to the solvent after it dissociates from the heme [1]. The ligand dissociation and transfer process in carboxy-myoglobin (MbCO) has been studied in a variety of experiments. In pump-probe experiments, a visible pump pulse at $580 \mathrm{~nm}$ ( $Q$ band) or at $400 \mathrm{~nm}$ (Soret band) induces a $\pi-\pi^{*}$ transition in the heme which results in dissociation of the $\mathrm{Fe}-\mathrm{CO}$ bond. A visible probe pulse then measures the electronic transition changes of the heme related to the $\mathrm{CO}$ dissociation [2]. A probe in the midinfrared range, on the other hand, is directly sensitive to the $\mathrm{CO}$ vibrational frequency changes. Carboxy-myoglobin (or -hemoglobin) dynamics upon ligand dissociation have previously been studied in the infrared [1,3-5] (IR). These experiments focused either on the bound $\mathrm{CO}$ or on $\mathrm{CO}$ at the docking site. Apart from the main vibrational absorption line $\left(A_{1}\right)$ of bound $\mathrm{CO}$, weaker absorption bands $\left(A_{0}, A_{3}\right)$ have been identified and attributed to different protein substates or conformations [6]. Two vibrational peaks $\left(B_{1}, B_{2}\right)$ were also observed at the docking site and attributed to two different orientations of the CO corresponding to a $180^{\circ}$ rotation [1]. CO dissociation has also been studied through its effect on heme vibrational modes involved in the transfer $[7,8]$.
Despite the extensive amount of data available on the ligand dissociation and transfer in $\mathrm{MbCO}$, very little is known about the very first steps during and immediately after ligand dissociation. We have chosen fs visible pump, mid-IR probe experiments to study the initial steps of the transfer process because the stretching frequency of $\mathrm{CO}$ is highly sensitive to $\mathrm{CO}$ ligation and to the local electric field. The probe central frequency was tuned from below the initial vibrational frequency of heme-bound $\mathrm{CO}$ $\left(1945 \mathrm{~cm}^{-1}\right)$ [4], to beyond the CO frequency at the docking site $\left(2130 \mathrm{~cm}^{-1}\right)$ [1] (see Fig. 1). In contrast to previous experiments, the differential transmission (DT) was not spectrally resolved. Therefore, we are not sensitive to perturbed polarization effects which occur at negative pump-probe delays: the probe induces a polarization that is modified by the arrival of the pump. In the frequency domain, i.e., in spectrally

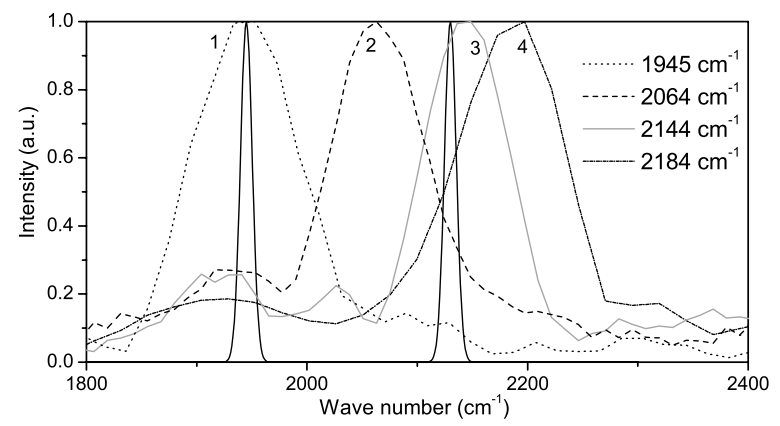

FIG. 1. Spectra of the different probes used. Probe 1 (3), dotted (solid grey) line, is tuned to the initial (final) vibrational frequency of $\mathrm{CO}$ bound to the heme ( $\mathrm{CO}$ at the docking site), respectively. Probe 2 (dashed line) is tuned to an intermediate frequency, and probe 4 (solid black line) is tuned to the highenergy side of the $\mathrm{CO}$ frequency at the docking site. Also shown are the bound $\mathrm{CO}$ and docking site absorption lines used in our calculations with maximum absorbances normalized to 1 . 
resolved experiments, this translates into spectral oscillations [9]. Therefore, the interpretation of spectrally resolved experiments is complicated when the dynamics under study is faster than the dephasing time.

DT experiments in the visible have shown that, seen from the heme, the photodissociation process is ultrafast $(<50 \mathrm{fs})$ [2]. Therefore, the absorption strength and frequency change of the $\mathrm{CO}$ vibrational transition from its heme-bound to its docking-site value has been implicitly assumed quasi-instantaneous until now. In this work, we demonstrate by contrast that the $\mathrm{CO}$ absorption strength changes gradually as it moves from the heme to the docking site. The dynamics of the $\mathrm{CO}$ vibrational absorption strength can thus be seen as a probe of the ligand dissociation and transfer. Density-functional calculations are in agreement with the finding that absorption strength and vibrational frequency changes are not linearly coupled.

A $100-\mathrm{kHz}$ Ti:sapphire amplified laser (Coherent RegA) produces a spectral continuum and seeds an 800$\mathrm{nm}$ pumped optical parametric amplifier (OPA). Our IR probe is generated by difference frequency mixing the signal and idler OPA output in a 1-mm-thick type II $\mathrm{AgGaS}_{2}$ crystal [10]. The 400-nm pump is obtained by frequency doubling the residual $800-\mathrm{nm}$ OPA pump. Pump and probe were collinearly focused to 100 and $80 \mu \mathrm{m}$ full width at half maximum (FWHM), respectively.

We use horse heart $\mathrm{Mb}$ (Sigma) in a 50-mM Tris $\mathrm{HCl}$ buffer $(p \mathrm{H}=7.6)$. The samples $(10 \mathrm{mM}$ in $\mathrm{Mb})$ are reduced with sodium dithionite, exposed to 1-atm CO, and loaded between 2-mm-thick $\mathrm{CaF}_{2}$ windows using a $25-\mu \mathrm{m}$ spacer. The samples are simultaneously rotated and translated at $293 \mathrm{~K}$ to ensure that every pump pulse illuminates a new spot of MbCO. At each shot, $25 \%$ of the $\mathrm{Fe}-\mathrm{CO}$ bonds are broken. A laser-synchronized mechanical chopper modulates the pump beam at $50 \mathrm{kHz}$. The difference between the transmitted and a reference IR intensity is detected by two $\mathrm{HgCdTe}$ detectors using a lock-in amplifier. The $100-\mathrm{kHz}$ repetition rate allows sufficient averaging in a few hours. The low noise reached with this setup is a prerequisite for the detection of a spectrally integrated signal. Indeed, the spectral width of our probe pulses $\left[\approx 100 \mathrm{~cm}^{-1}\right.$ (see Fig. 1)] is much broader than the width of the bound $\mathrm{CO}$ absorption line $\left(\approx 12 \mathrm{~cm}^{-1}\right.$ ), thus resulting in $10^{-5}-10^{-4}$ transmission changes upon $\mathrm{CO}$ dissociation. The zero pump-probe delay was determined by the pump-probe free-carrier absorption signal in $\mathrm{GaAs}$ placed behind a $\mathrm{CaF}_{2}$ window (maximum error $\pm 100 \mathrm{fs}$ ). The derivative of the GaAs signal corresponds to a pump-probe cross correlation. Assuming equal pump and probe durations, we obtain FWHM durations of about $250 \mathrm{fs}$. In order to avoid a contribution to the signal due to rotation of the $\mathrm{CO}$ molecules from an angle nearly perpendicular to the heme plane for heme-bound $\mathrm{CO}$ to an angle nearly parallel to the heme [11] for docked CO, we used an angle between pump and probe polarizations equal to $54.7^{\circ}$ (magic angle).

For probe $1\left(1945 \mathrm{~cm}^{-1}\right)$, the absorption decreases due to the displacement of the $\mathrm{CO}$ band out of the probe spectrum, as expected. The absorption decrease closely follows the GaAs signal, indicating that the $\mathrm{CO}$ vibrational frequency changes on a time scale faster than our time resolution. This is in agreement with previous DT measurements in the visible with a $580-\mathrm{nm}$ pump that indicate a decay of the lowest-lying heme-CO excited state into an excited unligated heme state in less than $50 \mathrm{fs}$ [2]. Excitation of the heme-CO group with a 400-nm pump may require an additional internal conversion time. However, this time must be very short since experiments with a 580- and a 400-nm pump showed no additional delay in the photodissociation process for $400-\mathrm{nm}$ excitation [12].

For probe $3\left(2145 \mathrm{~cm}^{-1}\right)$, we expect an increase of absorption as the $\mathrm{CO}$ evolves to the docking site. We observe instead an intriguing signal that first rises and subsequently decreases to reach a small positive value. This final value is in agreement with the decrease in absorption strength of $\mathrm{CO}$ at the docking site with respect to heme-bound $\mathrm{CO}$ [13]. Our experimental ratio of 21 between the absolute values of the DT at 1.75 ps for probes 1 and 3 is in reasonable agreement with the reported ratio of 34 between the $A$ and $B$ state absorbance [4]. However, the maximum value of the signal at around $200 \mathrm{fs}$ is surprisingly large and can only be explained if the absorption strength of the $\mathrm{CO}$ vibration remains high at early times and decays to its final value at a slower rate than the rate of vibrational frequency change.

In order to exclude vibrational cooling as a possible origin of the absorption decrease, we estimated the vibrational state of the dissociated CO from the 0.03 - $\AA$ change in the $\mathrm{CO}$ equilibrium distance with respect to bound $\mathrm{CO}$ [14] by assuming that the displaced ground-state wave function is close to a quasiclassical state in a harmonicoscillator potential. We find the majority of the population in the ground state $(83 \%), 16 \%$ in the first and $1 \%$ in the second excited state, in agreement with the experimental value of $8 \%$ of unbound $\mathrm{CO}$ being produced in the first excited state [1]. In addition, vibrational cooling takes place on a much longer time scale (several $100 \mathrm{ps}$ [15]).

Probe $2\left(2064 \mathrm{~cm}^{-1}\right)$ and probe $4\left(2185 \mathrm{~cm}^{-1}\right)$ are centered symmetrically at frequencies below and above the $\mathrm{CO}$ frequency at the docking site. The signals for these two probes are very similar and show the same behavior as the signal for probe 3 , only a smaller amplitude. These two signals can be fully understood in terms of the nonzero overlap between the probe spectrum and the final CO vibrational absorption line (see Fig. 1). Experiments at other intermediate frequency values gave results in agreement with the above interpretations.

We verified that these signals are due to a transient state of the $\mathrm{CO}$ molecule and not to the heating of our aqueous solvent or other artifacts from the $\mathrm{CaF}_{2}$ windows or the 
solvent by performing control experiments in deoxy-myoglobin with probe 2 as well as in the buffer solution for several probe frequencies. No signal was observed in all of these cases.

In order to describe our data, we used a phenomenological model for the $\mathrm{CO}$ oscillator with a Gaussian line shape and the following time-dependent parameters: central frequency, $\omega=2 \pi \sigma c$, absorption strength, $|\mu|^{2}$, and orientation given by the angle between the $\mathrm{CO}$ and the normal to the heme plane. The inverse linewidth $T=$ $[(\sqrt{2 \ln 2}) /(\pi \Delta \sigma c)]$ is kept constant. The pump-induced changes of these parameters are included in a nonstationary susceptibility,

$$
\mathcal{R}\left(t, t^{\prime}\right) \propto\left\langle\mu(t) \mu\left(t^{\prime}\right)\right\rangle e^{-i \int_{t^{\prime}}^{t} d s \omega(s)} e^{-\left(t-t^{\prime}\right)^{2} / 2 T^{2}},
$$

which relates the induced polarization $\mathcal{P}(t)$ to the complex incident IR electric field $\mathcal{E}(t): \quad \mathcal{P}(t)=$ $\int_{-\infty}^{t} d t^{\prime} \mathcal{R}\left(t, t^{\prime}\right) \mathcal{E}\left(t^{\prime}\right)$. The absorption, $A$, is then derived from $A \propto \operatorname{Im} \int_{-\infty}^{\infty} d t \mathcal{E}^{\star}(t) \mathcal{P}(t)$ [16] within the rotating wave approximation. The parentheses denote a weighted average over molecular orientations. Because of the weakness of the other $A$ substates[4], we considered only the main heme-bound vibrational line $A_{1}$ as a Gaussian centered at $1945 \mathrm{~cm}^{-1}$ with a FWHM of $12 \mathrm{~cm}^{-1}$ [4]. In order to limit the number of fit parameters, we used only one final $\mathrm{CO}$ state centered at $2130 \mathrm{~cm}^{-1}$ with a FWHM of $12 \mathrm{~cm}^{-1}$.

We used a quasi-instantaneous vibrational frequency change and an absorption strength that changes at a slower rate between the initial and the final value (34 times smaller [4]) to reproduce the experiment. The calculated signal is normalized to the signal for probe 1 at large pump-probe delays. Good qualitative agreement with the experiment is obtained for an exponential decay time of the absorption strength of 400 fs (solid lines in Fig. 2).

The fact that the two main parameters of the $\mathrm{CO}$ vibrator, frequency and absorption strength, should change at different rates may seem surprising. However, the use of a quasi-instantaneous change both for the vibrational frequency and the absorption strength cannot reproduce the amplitude of the data for probes 2 to 4 (Fig. 2, dashed lines). On the other hand, the use of a gradual vibrational frequency change with the same rate as that of the absorption strength change shifts the maxima of the signals calculated for probes 3 and 4 to larger delays times, decreases their amplitude, and deteriorates the agreement with the experiment. We also verified that the small chirp present in our pulses has little influence on the calculated signals.

In order to gain insight into the relationship between frequency and absorption strength changes of the $\mathrm{CO}$, we performed density-functional (DFT) calculations for a model system consisting of an iron porphine with a bound CO perpendicular to the porphine, an axial imidazole, and a distal imidazole hydrogen bonded to the oxygen of

018102-3

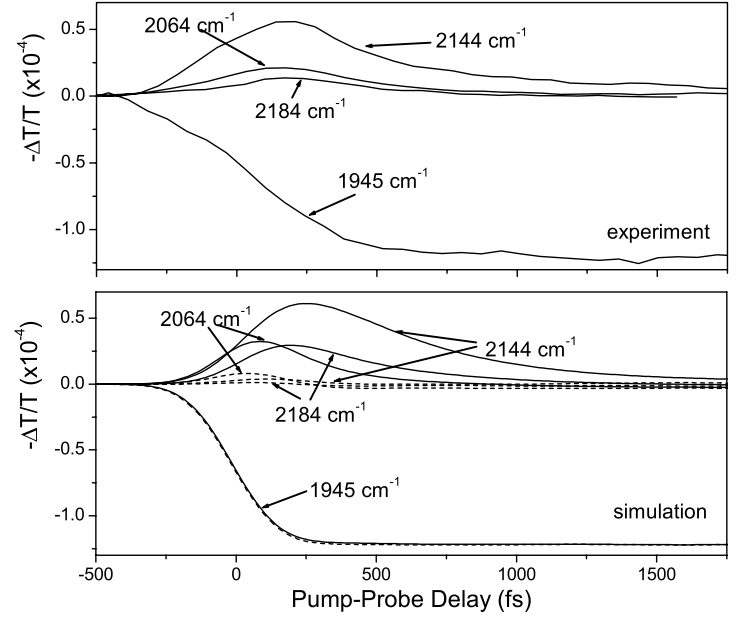

FIG. 2. Differential transmission in MbCO for the four different probes of Fig. 1. Upper panel: Experiment. Pump energies of 180-280 nJ were used. The DT signals were linearly scaled to the average pump energy of $220 \mathrm{~nJ}$. The relative amplitudes are correct within approximately 20\%. Lower panel: Calculated signals using a phenomenological model that assume a progressive (quasi-instantaneous) absorption strength change for the $\mathrm{CO}$ vibration are shown as solid (dashed) lines.

$\mathrm{CO}$ (with a Fe-O ... H angle of $110^{\circ}$ and a $\mathrm{O} \ldots \mathrm{H}$ distance of $1.92 \AA$; for details, see Ref. [14]). The two imidazoles simulate the histidine residues His93 and His64, respectively. Starting with the fully geometry optimized structure, the $\mathrm{Fe}-\mathrm{C}$ distance was increased and the $\mathrm{C}-\mathrm{O}$ bond length alone was allowed to reoptimize with all other coordinates held fixed. The $\mathrm{CO}$ vibrational frequency and absorption intensity as a function of Fe-C distance are shown in Fig. 3. The most striking feature is that the intensity does not decrease as quickly as the frequency increases, in general agreement with the conclusion drawn from the experiment and from the comparison with the phenomenological model. Crudely speaking, we can associate the intensity with the amount of charge displacement from the iron onto the $\mathrm{CO}$ and the frequency with the $\mathrm{CO}$ bond length [14]. The DFT calculations show a charge displacement from the iron to $\mathrm{CO}$ that affects the

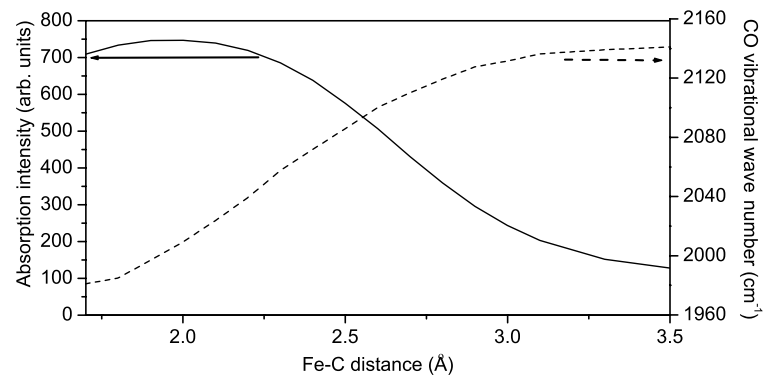

FIG. 3. Density-functional calculations of the $\mathrm{CO}$ vibrational absorption intensity (solid line) and frequency (dashed line) using a model system consisting of an iron porphine with a bound $\mathrm{CO}$, an axial and a distal imidazole (see text). 
intensity of the $\mathrm{CO}$ in a manner that is not proportionally coupled to frequency [17]. The calculated absorption change is smaller than the experimental one. This is not surprising given the approximations made: the spin change of the iron from $S=0$ to $S=2$ after ligand photodissociation [2], the $\mathrm{CO}$ orientation change, and the full heme-pocket environment responsible for a docking-site absorbance smaller than that of free $\mathrm{CO}$ in the gas state [18] were not taken into account.

It may be questioned why this progressive change in absorption strength has not been reported previously. The spectrally resolved experiments of Ref. [1] showed a narrow band absorption and a broad underlying band [4] that was subtracted away. As these authors point out, the integrated absorbance of the narrow band $B$ states shown in Ref. [1] does not take into account the total absorption intensity of the $\mathrm{CO}$ vibrational transition. Thus, it is not surprising that our signal, which integrates over the narrow band and broadened features, shows different behavior. The 9-fold absorption decrease observed here and the 2-fold increase of the narrow band absorption observed previously [1] imply that the spectrally integrated contribution of the broad underlying band must be about 18 times larger than that of the narrow band at 200 fs. Given the different widths of these contributions, however, the maximum of the underlying absorption needs to be only 3 times larger than the narrow band peak absorption. Several effects can give rise to a transient broadening of the $\mathrm{CO}$ vibrational absorption: the transient rotational freedom of the $\mathrm{CO}$ before its motion becomes constrained at the docking site $[1,4]$, the inhomogeneity effect related to the different trajectories taken by different $\mathrm{CO}$ molecules as well as to different conformational substates of the protein, and an inherent broadening effect due to the decrease of the absorption strength. This decrease with a characteristic time of $400 \mathrm{fs}$ contributes to the polarization decrease in the time domain and thus leads to a broadening in the spectral domain. The rotation of the $\mathrm{CO}$ dipole (from perpendicular to parallel to the heme), which takes place on the same time scale [1], also contributes to a polarization decrease and thus participates in the spectral broadening. Our spectrally integrated experiments are not sensitive to these broadening effects as long as the total spectral width of the $\mathrm{CO}$ absorption remains narrower than the probe width and are therefore ideal for a measurement of the total absorption strength. Calculations with our phenomenological model show that a transient broadening of the $\mathrm{CO}$ absorption up to a FWHM of $40 \mathrm{~cm}^{-1}$ practically does not modify the signals.

In conclusion, our experiments allow the assessment of a gradual change in absorption strength between the heme-bound and the docking-site values. Based on density-functional calculations we attribute this effect to charge displacement from the heme iron to $\mathrm{CO}$ which affects the absorption strength in a manner nonlinearly coupled to the vibrational frequency. To our knowledge, this is the first observation of a progressive change in absorption strength following dissociation of a bond. This may well be a more general feature in bond-breaking reactions in ligated metals. In the case of CO-ligated heme proteins, this implies that the $\mathrm{CO}$ vibrational absorption strength can be used as a probe of the ligand environment during the dissociation and transfer process.

We are grateful to M. Bierry and X. Solinas for the realization of the $50-\mathrm{kHz}$ synchronized chopper.

[1] M. Lim, T. A. Jackson, and P. A. Anfinrud, Nat. Struct. Biol. 4, 209 (1997).

[2] J.-L. Martin, A. Migus, C. Poyart, Y. Lecarpentier, R. Astier, and A. Antonetti, Proc. Natl. Acad. Sci. U.S.A. 80, 173 (1983); J.W. Petrich, C. Poyart, and J.-L. Martin, Biochemistry 27, $4049 \quad$ (1988); S. Franzen, L. Kiger, C. Poyart, and J.-L. Martin, Biophys. J. 80, 2372 (2001).

[3] T. P. Causgrove and R. Brian Dyer, J. Phys. Chem. 100, 3273 (1996).

[4] M. Lim, T. A. Jackson, and P. A. Anfinrud, J. Chem. Phys. 102, 4355 (1995)

[5] P. A. Anfinrud, C. Han, and R. M. Hochstrasser, Proc. Natl. Acad. Sci. U.S.A. 86, 8387 (1989).

[6] H. Frauenfelder, S. G. Sligar, and P. G. Wolynes, Science 254, 1598 (1991).

[7] M.-L. Groot, M. H. Vos, I. Schlichting, F. Van Mourik, M. Joffre, J.-C Lambry, and J.-L. Martin, Proc. Natl. Acad. Sci. U.S.A. 99, 1323 (2002).

[8] L. Y. Zhu, J.T. Sage, and P. M. Champion, Science 266, 629 (1994); M. R. Armstrong, J. P. Ogilvie, M. L. Cowan, A. M. Nagy, and R. J. D. Miller, Proc. Natl. Acad. Sci. U.S.A. 100, 4990 (2003).

[9] M. Joffre et al., Opt. Lett. 13, 276 (1988); P. Hamm, Chem. Phys. 200, 415 (1995).

[10] M. K. Reed and M. K. Steiner Shepard, IEEE J. Quantum Electron. 32, 1273 (1996); B. Golubovic and M. K. Reed, Opt. Lett. 23, 1760 (1998).

[11] M. Lim, T. A. Jackson, and P. A. Anfinrud, Science 269, 962 (1995)

[12] X. Ye, A. Demidov, and P. M. Champion, J. Am. Chem. Soc. 124, 5914 (2002).

[13] The larger absorption strength for heme-bound $\mathrm{CO}$ is related to charge injection from the iron.

[14] S. Franzen, J. Am. Chem. Soc. 124, 13271 (2002).

[15] D. E. Sagnella, J. E. Straub, T. A. Jackson, M. Lim, and P. A. Anfinrud, Proc. Natl. Acad. Sci. U.S.A. 96, 14324 (1999).

[16] S. Mukamel, Principles of Nonlinear Optical Spectroscopy (Oxford University Press, Oxford, 1995).

[17] A charge displacement was also observed in recent calculations of the $Q$-band excited heme-CO states as a function of Fe-C distance: B. D. Dunietz, A. Dreuw, and M. Head-Gordon, J. Phys. Chem. B 107, 5623 (2003).

[18] J. O. Alben, D. Beece, S.F. Bowne, W. Doster, L. Eisenstein, H. Fraunfelder, D. Good, J. D. McDonald, M. C. Marden, P. P. Moh, L. Reinisch, A. H. Reynolds, E. Shyamsunder, and K.T. Yue, Proc. Natl. Acad. Sci. U.S.A. 79, 3744 (1982). 